\title{
Fluctuations from dissipation in a hot non-Abelian plasma
}

\author{
Daniel F. Litim* \\ Institut für Theoretische Physik, Philosophenweg 16, D-69120 Heidelberg, Germany \\ Cristina Manuel ${ }^{\dagger}$ \\ Theory Division, CERN, CH-1211 Geneva 23, Switzerland \\ (Received 29 November 1999; published 10 May 2000)
}

\begin{abstract}
We consider a transport equation of the Boltzmann-Langevin type for non-Abelian plasmas close to equilibrium to derive the spectral functions of the underlying microscopic fluctuations from the entropy. The correlator of the stochastic source is obtained from the dissipative processes in the plasma. This approach, based on classical transport theory, exploits the well-known link between a linearized collision integral, the entropy and the spectral functions. Applied to the ultra-soft modes of a hot non-Abelian (classical or quantum) plasma, the resulting spectral functions agree with earlier findings obtained from the microscopic theory. As a by-product, it follows that Bödeker's effective theory is consistent with the fluctuation-dissipation theorem.
\end{abstract}

PACS number(s): 12.38.Mh

It has been recognized that dynamical properties of (nonperturbative) quasi-particle excitations in non-Abelian plasmas can be described very efficiently by means of effective transport equations. A prominent recent example is given by Bödeker's effective classical theory for the ultra-soft modes in a hot non-Abelian plasma close to equilibrium [1], which corresponds to a transport equation of the BoltzmannLangevin type. In [2], a general procedure has been presented, based on classical colored point particles, to obtain effective transport equations from the microscopic theory after integrating-out the fluctuations about the mean values, and taking the Gibbs ensemble average in phase space. On the one-loop level, the same mean field equations of [2] have been obtained recently within a many-particle world line formalism [3] (see also [4]). The collision integral and the source of stochastic noise of [1] have been obtained from [2] to leading order in a weak coupling expansion, and at logarithmic accuracy. It was also realized that the dynamical equations are the same for the classical and the quantum plasma, changing only in the value of the Debye mass [2]. Other approaches to obtain the collision term of [1] have been reported as well [5-7].

In the present article we consider, based on classical transport theory, a generic Boltzmann-Langevin equation for the one-particle distribution function $f(x, p, Q)$, given as

$$
\begin{aligned}
& p^{\mu}\left(\frac{\partial}{\partial x^{\mu}}-g f^{a b c} A_{\mu}^{b} Q^{c} \frac{\partial}{\partial Q^{a}}-g Q_{a} F_{\mu \nu}^{a} \frac{\partial}{\partial p_{\nu}}\right) f(x, p, Q) \\
& \quad=C[f](x, p, Q)+\zeta(x, p, Q) .
\end{aligned}
$$

Here, the variables $Q$ describe the non-Abelian color charges. The transport equation contains an effective collision term $C[f]$ and an associated source for stochastic noise $\zeta$. The $\operatorname{SU}(N)$ gauge fields appearing in the above equation

\footnotetext{
*Email address: D.Litim@thphys.uni-heidelberg.de

†Email address: Cristina.Manuel@cern.ch
}

are self-consistent, that is, generated by the same particles of the plasma. The Yang-Mills equation are

$$
\left(D_{\mu} F^{\mu \nu}\right)_{a}=J_{a}^{\nu}(x) \equiv g \sum_{\substack{\text { helicities } \\ \text { species }}} \int d P d Q Q_{a} p^{\nu} f(x, p, Q),
$$

where the momemtum measure reads $d P$ $=d^{4} p 2 \Theta\left(p_{0}\right) \delta\left(p^{2}-m^{2}\right)$, and the color measure $d Q$ was defined in [2]. The current in Eq. (2) is covariantly conserved. We work in natural units $c=\hbar=k_{B}=1$, unless otherwise specified. From now on we will omit the sum over different species of particles and helicities.

In the collisionless limit $C=\zeta=0$, the above set of transport equation reduces to those introduced by Heinz [8]. In the general case however, the right-hand side (RHS) of Eq. (1) does not vanish due to effective interactions (collisions) in the plasma, resulting in the term $C[f]$. In writing Eq. (1), we have already made the assumption that the one-particle distribution function $f$ is a fluctuating quantity. This is quite natural having in mind that $f$ describes a "coarse-grained" microscopic distribution function for colored point particles, and justifies the presence of the stochastic source $\zeta$ in the transport equation. For non-charged particles, a similar kinetic equation has already been considered in [9] (see also [10], where stochastic noise is introduced to a SchwingerDyson approach).

Given the stochastic dynamical equation (1), the question raises as to what can be said on general grounds about the spectral functions of $f$ and $\zeta$. Here, we shall assume that the dissipative processes are known close to equilibrium, but no further information is given regarding the underlying fluctuations. This way of proceeding is complementary to [2], where the RHS of Eq. (1) has been obtained from correlators of the microscopic statistical fluctuations. We then show that the spectral function of the fluctuations and the noise correlator close to equilibrium can be obtained from the knowledge of the entropy of the plasma, and from the dissipative term in the effective transport equation. This gives a welldefined prescription as to how the correct source for noise 
can be identified without the detailed knowledge of the underlying microscopic dynamics responsible for the dissipation. The basic idea behind this approach relies on the essence of the fluctuation-dissipation theorem (FDT). While this theorem is more general, here we will only discuss the close to equilibrium situations. According to the FDT if a fluctuating system remains close to equilibrium, then the dissipative process occurring in it are known. Vice versa, if one knows the dissipative process in the system, one can describe the fluctuations without an explicit knowledge of the microscopic structure or processes in the system. The cornerstone of our approach is the entropy of the fluctuating system, which serves to identify the thermodynamical forces, and leads to the spectral function for the deviations from the non-interacting equilibrium.

Before entering into the discussion of plasmas, we will illustrate this way of proceeding by reviewing the simplest setting of classical linear dissipative systems [11]. A generalization to the more complex case of non-Abelian plasmas will then become a natural step to perform. We consider a classical homogeneous system described by a set of variables $x_{i}$, where $i$ is a discrete index running from 1 to $n$. These variables are normalized in such a way that their mean values at equilibrium vanish. The entropy of the system is a function of the quantities $x_{i}, S\left(x_{i}\right)$. If the system is at equilibrium, the entropy reaches its maximum, and thus $\left(\partial S / \partial x_{i}\right)_{\mathrm{eq}}=0, \forall i$. If the system is taken slightly away from equilibrium, then one can expand the difference $\Delta S=S$ $-S_{\text {eq }}$, where $S_{\text {eq }}$ is the entropy at equilibrium, in powers of $x_{i}$. If we expand up to quadratic order, then

$$
\Delta S=\frac{1}{2}\left(\frac{\partial^{2} S}{\partial x_{i} \partial x_{j}}\right)_{\mathrm{eq}} x^{i} x^{j} \equiv-\frac{1}{2} \beta_{i j} x^{i} x^{j} .
$$

The matrix $\beta_{i j}$ is symmetric and positive-definite, since the entropy reaches a maximum at equilibrium. The thermodynamic forces $F_{i}$ are defined as the gradients of $\Delta S$

$$
F_{i}=-\frac{\partial \Delta S}{\partial x_{i}} .
$$

For a system close to equilibrium the thermodynamic forces are linear functions of $x_{i}, F_{i}=\beta_{i j} x^{j}$. If the system is at equilibrium, the thermodynamic forces vanish. In more general situations the variables $x_{i}$ will evolve in time. The time evolution of these variables is given as functions of the thermodynamical forces. In a close to equilibrium case one can expect that the evolution is linear in the forces

$$
\frac{d x^{i}}{d t}=-\gamma^{i j} F_{j}+\zeta^{i},
$$

which, in turn, can be expressed as

$$
\frac{d x^{i}}{d t}=-\lambda^{i j} x_{j}+\zeta^{i}
$$

The first term in the RHS of the above equation describes the mean regression of the system towards equilibrium, while the second term is the source for stochastic noise. The quantities $\gamma^{i j}$ are known as the kinetic coefficients, and it is not difficult to check that $\gamma_{i j}=\lambda_{i k} \beta_{k j}^{-1}$. From the value of the coefficients $\beta_{i j}$ one can deduce the equal time correlator

$$
\left\langle x_{i}(t) x_{j}(t)\right\rangle=\beta_{i j}^{-1},
$$

which is used to obtain Einstein's law

$$
\left\langle x^{i}(t) F_{j}(t)\right\rangle=\delta_{j}^{i} .
$$

After taking the time derivative of Eq. (8), assuming that the noise is white and Gaussian

$$
\left\langle\zeta^{i}(t) \zeta^{j}\left(t^{\prime}\right)\right\rangle=\nu^{i j} \delta\left(t-t^{\prime}\right),
$$

we find that the strength of the noise self-correlator $\nu$ is determined by the dissipative process

$$
\nu^{i j}=\gamma^{i j}+\gamma^{j i}
$$

which is the FDT relation we have been aiming at.

We now come back to the case of a non-Abelian plasma and generalize the above discussion to the case of our concern. We will consider the non-Abelian plasma as a linear dissipative system, assuming that we know the collision term in the transport equation. In order to adopt the previous reasoning, we have to identify the dissipative term in the transport equation, and to express it as a function of the thermodynamical force obtained from the entropy. The deviation from the equilibrium distribution is given here by

$$
\Delta f(x, p, Q)=f(x, p, Q)-f_{\text {eq }}\left(p_{0}\right),
$$

and replaces the variables $x_{i}$ discussed above. The entropy flux density for classical plasmas is given as

$$
S_{\mu}(x)=-\int d P d Q p_{\mu} f(x, p, Q)\left(\ln \left[f(x, p, Q) h^{3}\right]-1\right),
$$

where $h$ is an arbitrary constant such that $f(x, p, Q) h^{3}$ is dimensionless. The $\mu=0$ component of Eq. (12) gives the entropy density of the system. The entropy itself is then obtained as $S=\int d^{3} x S_{0}(x)$.

We shall now assume that the deviation of the mean particle number from the equilibrium one is small within a coarse-graining volume. This can always be arranged for at small gauge coupling ${ }^{1} g \ll 1$. We then obtain $\Delta S$ just by expanding the expression of the entropy density in powers of $\Delta f$ up to quadratic order. It is important to take into account that we will consider situations where the small deviations

\footnotetext{
${ }^{1}$ More precisely, in the close-to-equilibrium plasma we consider $f$ as being coarse-grained over a Debye volume. This entails that (i) the particle number fluctuations within a Debye volume are parametrically suppressed by powers of the plasma parameter (that is, ultimately, by $g$ ), (ii) the two- and higher-particle distribution functions are suppressed as opposed to $f$, and (iii) typical relaxation scales or times are not affected by the coarse-graining.
} 
from equilibrium are such that both the particle number and the energy flux remain constant, thus

$$
\int d P d Q \Phi(p) \Delta f(x, p, Q)=0 \text { for } \Phi(p)=p_{0}, p_{0} p_{\mu} .
$$

Under those assumptions, one reaches to

$$
\begin{aligned}
\Delta S_{0}(x) & =-\int d P d Q p_{0} \frac{(\Delta f(x, p, Q))^{2}}{2 f_{\mathrm{eq}}\left(p_{0}\right)} \\
& =-\int d^{3} p d Q \frac{(\Delta f(x, \mathbf{p}, Q))^{2}}{2 f_{\mathrm{eq}}\left(\omega_{p}\right)},
\end{aligned}
$$

where in the last equality we have taken into account the mass-shell condition, with $p_{0}=\omega_{p}=\sqrt{\mathbf{p}^{2}+m^{2}}$. Without loss of generality, we will consider from now on the case of massless particles, so $\omega_{p}=p=|\mathbf{p}|$.

The thermodynamic force associated to $\Delta f$ is defined from the entropy as

$$
F(x, \mathbf{p}, Q)=-\frac{\delta \Delta S}{\delta \Delta f(x, \mathbf{p}, Q)}=\frac{\Delta f(x, \mathbf{p}, Q)}{f_{\mathrm{eq}}(p)} .
$$

We now linearize the transport equation (1) and express the collision integral close to equilibrium in terms of the thermodynamical force. Dividing Eq. (1) by $p_{0}$ and imposing the mass-shell constraint, we find

$$
v^{\mu} D_{\mu} \Delta f-g v^{\mu} Q_{a} F_{\mu 0}^{a} \frac{d f_{\mathrm{eq}}}{d p}=C[\Delta f](x, \mathbf{p}, Q)+\zeta(x, \mathbf{p}, Q),
$$

where $v^{\mu}=p^{\mu} / p_{0}=(1, \mathbf{v})$, with $\mathbf{v}^{2}=1$. We also introduced the shorthand $D_{\mu} \Delta f \equiv\left(\partial_{\mu}-g f^{a b c} A_{\mu, b} Q_{c} \partial_{a}^{Q}\right) \Delta f$ [2]. It is understood that the collision integral has been linearized, and we write it as

$$
\begin{aligned}
C[\Delta f](t, \mathbf{x}, \mathbf{p}, Q)= & \int d^{3} x^{\prime} d^{3} p^{\prime} d Q^{\prime} K\left(\mathbf{x}, \mathbf{p}, Q ; \mathbf{x}^{\prime}, \mathbf{p}^{\prime}, Q^{\prime}\right) \\
& \times \Delta f\left(t, \mathbf{x}^{\prime}, \mathbf{p}^{\prime}, Q^{\prime}\right),
\end{aligned}
$$

with $t \equiv x_{0}$. For simplicity, we take the collision integral local in time, but unrestricted elsewise. ${ }^{2}$ According to the FDT, the source of stochastic noise has to obey

$$
\begin{aligned}
& \left\langle\zeta(x, \mathbf{p}, Q) \zeta\left(x^{\prime}, \mathbf{p}^{\prime}, Q^{\prime}\right)\right\rangle \\
& \quad=-\left(\frac{\delta C[F](x, \mathbf{p}, Q)}{\delta F\left(x^{\prime}, \mathbf{p}^{\prime}, Q^{\prime}\right)}+\frac{\delta C[F]\left(x^{\prime}, \mathbf{p}^{\prime}, Q^{\prime}\right)}{\delta F(x, \mathbf{p}, Q)}\right)
\end{aligned}
$$

\footnotetext{
${ }^{2}$ Of course, gauge invariance imposes further conditions on both the collision term and the noise. However, these constraints are of no relevance for the present discussion.
}

in full analogy to Eq. (10). With the knowledge of the thermodynamical force (15) and the linearized collision term (17) we arrive at

$$
\begin{aligned}
& \left\langle\zeta(x, \mathbf{p}, Q) \zeta\left(x^{\prime}, \mathbf{p}^{\prime}, Q^{\prime}\right)\right\rangle \\
& \quad=-\left(f_{\text {eq }}(p) K\left(\mathbf{x}, \mathbf{p}, Q ; \mathbf{x}^{\prime}, \mathbf{p}^{\prime}, Q^{\prime}\right)+\operatorname{sym} .\right) \delta\left(t-t^{\prime}\right) .
\end{aligned}
$$

Here, symmetrization in $(\mathbf{x}, \mathbf{p}, Q) \leftrightarrow\left(\mathbf{x}^{\prime}, \mathbf{p}^{\prime}, Q^{\prime}\right)$ is understood.

Notice that we can derive the equal time correlator for the deviations from the equilibrium distribution simply from the knowledge of the entropy and the thermodynamical force, exploiting Einstein's law in full analogy to the corresponding relation (7). Using Eq. (15) we find

$$
\begin{aligned}
& \left\langle\Delta f(x, \mathbf{p}, Q) \Delta f\left(x^{\prime}, \mathbf{p}^{\prime}, Q^{\prime}\right)\right\rangle_{t=t^{\prime}} \\
& \quad=f_{\mathrm{eq}}(p) \delta^{(3)}\left(\mathbf{x}-\mathbf{x}^{\prime}\right) \delta^{(3)}\left(\mathbf{p}-\mathbf{p}^{\prime}\right) \delta\left(Q-Q^{\prime}\right) .
\end{aligned}
$$

If the fluctuations $\Delta f$ have vanishing mean value, then Eq. (20) reproduces the well-known result that the correlator of fluctuations at equilibrium is given by the equilibrium distribution function. In order to make contact with the results of [2], we go a step further and consider the case where $\Delta f$ has a non-vanishing mean value to leading order in the gauge coupling. Splitting $\Delta f=g \bar{f}^{(1)}+\delta f$ into a deviation of the mean part $\langle\Delta f\rangle=g \bar{f}^{(1)}$ and a fluctuating part $\langle\delta f\rangle=0$ and using Eq. (20), we obtain the equal time correlator for the fluctuations $\delta f$ as

$$
\begin{aligned}
\left\langle\delta f(x, \mathbf{p}, Q) \delta f\left(x^{\prime}, \mathbf{p}^{\prime}, Q^{\prime}\right)\right\rangle_{t=t^{\prime}} \\
=f_{\mathrm{eq}}(p) \delta^{(3)}\left(\mathbf{x}-\mathbf{x}^{\prime}\right) \delta^{(3)}\left(\mathbf{p}-\mathbf{p}^{\prime}\right) \delta\left(Q-Q^{\prime}\right) \\
\quad-\left.g^{2} \bar{f}^{(1)}(x, \mathbf{p}, Q) \bar{f}^{(1)}\left(x^{\prime}, \mathbf{p}^{\prime}, Q^{\prime}\right)\right|_{t=t^{\prime}} .
\end{aligned}
$$

This result agrees with the correlator obtained in [2] from the Gibbs ensemble average as defined in phase space in the limit where two-particle correlations are small and given by products of one-particle correlators.

Up to now we have dealt with purely classical plasmas. On the same footing, we can consider the soft and ultra-soft modes in a hot quantum plasma. These can be treated classically as their occupation numbers are large. The sole effect from their quantum nature reduces to the different statistics, Bose-Einstein or Fermi-Dirac as opposed to MaxwellBoltzmann. The corresponding quantum FDT reduces to an effective classical one $[11,12]$.

Some few changes are necessary to study hot quantum plasmas. As in [2], we change the normalization of $f$ by a factor of $(2 \pi \hbar)^{3}$ to obtain the standard normalization for the (dimensionless) quantum distribution function. Thus, the momentum measure is also modified by the same factor, $d P=d^{4} p 2 \Theta\left(p_{0}\right) \delta\left(p^{2}\right) /(2 \pi \hbar)^{3}$ for massless particles, and $\hbar=1$. To check the FDT relation in this case one needs to 
start with the correct expression for the entropy for a quantum plasma. The entropy flux density, as a function of $f(x, p, Q)$, is given by

$$
S_{\mu}(x)=-\int d P d Q p_{\mu}(f \ln f \mp(1 \pm f) \ln (1 \pm f)),
$$

where the upper or lower sign applies for bosons or fermions. From the above expression of the entropy one can compute $\Delta S$, and proceed exactly as in the classical case, expanding the entropy up to quadratic order in the deviations from equilibrium. Thus, we obtain the noise correlator

$$
\begin{aligned}
& \left\langle\zeta(x, \mathbf{p}, Q) \zeta\left(x^{\prime}, \mathbf{p}^{\prime}, Q^{\prime}\right)\right\rangle \\
& =-(2 \pi)^{3}\left(f_{\mathrm{eq}}(p)\left[1 \pm f_{\mathrm{eq}}(p)\right]\right. \\
& \left.\quad \times K\left(\mathbf{x}, \mathbf{p}, Q ; \mathbf{x}^{\prime}, \mathbf{p}^{\prime}, Q^{\prime}\right)+\operatorname{sym} .\right) \delta\left(t-t^{\prime}\right) .
\end{aligned}
$$

Again, the spectral functions of the deviations from equilibrium are directly deduced from the entropy. As a result, we find

$$
\begin{aligned}
\langle\Delta f(x, & \left.\mathbf{p}, Q) \Delta f\left(x^{\prime}, \mathbf{p}^{\prime}, Q^{\prime}\right)\right\rangle_{t=t^{\prime}} \\
= & (2 \pi)^{3} f_{\mathrm{eq}}(p)\left(1 \pm f_{\mathrm{eq}}(p)\right) \delta^{(3)}\left(\mathbf{x}-\mathbf{x}^{\prime}\right) \\
& \times \delta^{(3)}\left(\mathbf{p}-\mathbf{p}^{\prime}\right) \delta\left(Q-Q^{\prime}\right) .
\end{aligned}
$$

Expanding $\Delta f=g \bar{f}^{(1)}+\delta f$ as above, we obtain the equal time correlator for $\delta f$, which agrees with the findings of [2] in the case where two-particle distribution functions can be expressed as products of one-particle distributions.

With the knowledge of the above spectral functions for the fluctuations in a classical or quantum plasma one can derive further spectral distributions for different physical quantities. In particular, we can find the correlations of the self-consistent gauge field fluctuations once the basic correlators as given above are known. This is how those spectral functions were deduced in [2].

As a particular example of the above we consider Bödeker's effective kinetic equations which couple to the ultrasoft gauge field modes with spatial extensions $\gg 1 / m_{D}$, where $m_{D}$ is the Debye mass in a non-Abelian plasma close to equilibrium. The linearized collision integral has been obtained to leading logarithmic accuracy by several different approaches [1,2,5-7]. To leading order, they all employ an IR cutoff of the order of $g m_{D}$ for the elsewise unscreened magnetic sector.

We will first concentrate on the classical plasma, for particles carrying two helicities. It is most efficient to write the transport equation not in terms of the full one-particle distribution function, but in terms of the current density

$$
\mathcal{J}_{a}^{\rho}(x, \mathbf{v})=4 \pi g v^{\rho} \int d p d Q p^{2} Q_{a} \Delta f(x, \mathbf{p}, Q) .
$$

(Notice that $f_{\text {eq }}$ gives no contribution to the current.) The current of Eq. (2) follows after integrating over the angles of $\mathbf{v}, J_{a}^{\mu}(x)=\int(d \Omega / 4 \pi) \mathcal{J}_{a}^{\mu}(x, \mathbf{v})$ [2]. Expressed in terms of Eq. (25), the linearized Boltzmann-Langevin equation (16) becomes

$$
\begin{aligned}
{\left[v^{\mu} D_{\mu}, \mathcal{J}^{\rho}\right](x, \mathbf{v})=} & -m_{D}^{2} v^{\rho} v_{\mu} F^{\mu 0}(x) \\
& +v^{\rho} C\left[\mathcal{J}^{0}\right](x, \mathbf{v})+\zeta^{\rho}(x, \mathbf{v}),
\end{aligned}
$$

where $m_{D}$ is the Debye mass [2]

$$
m_{D}^{2}=-8 \pi g^{2} C_{2} \int d p p^{2} \frac{d f_{\mathrm{eq}}}{d p}
$$

and the constant $C_{2}$ depends on the representation of the colored particles

$$
\int d Q Q_{a} Q_{b}=C_{2} \delta_{a b}
$$

The linearized collision integral is related to Eq. (17) by

$$
\begin{aligned}
C\left[\mathcal{J}_{a}^{0}\right](x, \mathbf{v})= & 4 \pi g \int d^{3} x^{\prime} d \Omega_{\mathbf{v}^{\prime}} d p d p^{\prime} d Q \\
& \times d Q^{\prime} p^{2} p^{\prime 2} Q_{a} K\left(\mathbf{x}, \mathbf{p}, Q ; \mathbf{x}^{\prime}, \mathbf{p}^{\prime}, Q^{\prime}\right) \\
& \times \Delta f\left(t, \mathbf{x}^{\prime}, \mathbf{p}^{\prime}, Q^{\prime}\right)
\end{aligned}
$$

and has been obtained explicitly $[1,2,5-7]$ as

$$
C\left[\mathcal{J}_{a}^{0}\right](x, \mathbf{v})=-\gamma \int \frac{d \Omega_{\mathbf{v}^{\prime}}}{4 \pi} \mathcal{I}\left(\mathbf{v}, \mathbf{v}^{\prime}\right) \mathcal{J}_{a}^{0}\left(x, \mathbf{v}^{\prime}\right)
$$

where the kernel reads

$$
\mathcal{I}\left(\mathbf{v}, \mathbf{v}^{\prime}\right)=\delta^{(2)}\left(\mathbf{v}-\mathbf{v}^{\prime}\right)-\frac{4}{\pi} \frac{\left(\mathbf{v} \cdot \mathbf{v}^{\prime}\right)^{2}}{\sqrt{1-\left(\mathbf{v} \cdot \mathbf{v}^{\prime}\right)^{2}}}
$$

and $\gamma=g^{2} N T \ln (1 / g) / 4 \pi$. Comparing Eq. (29) with Eq. (30) we learn that only the part of the kernel $K$ which is symmetric under $(\mathbf{x}, \mathbf{p}, Q) \leftrightarrow\left(\mathbf{x}^{\prime}, \mathbf{p}^{\prime}, Q^{\prime}\right)$ contributes in the present case. This part can be expressed as

$$
\begin{aligned}
K\left(\mathbf{x}, \mathbf{p}, Q ; \mathbf{x}^{\prime}, \mathbf{p}^{\prime}, Q^{\prime}\right)= & -\gamma \frac{\mathcal{I}\left(\mathbf{v}, \mathbf{v}^{\prime}\right)}{4 \pi p^{2}} \delta\left(p-p^{\prime}\right) \\
& \times \delta\left(Q-Q^{\prime}\right) \delta^{(3)}\left(\mathbf{x}-\mathbf{x}^{\prime}\right) .
\end{aligned}
$$

According to our findings above, the self-correlator of the stochastic source for the classical plasma obeys

$$
\begin{aligned}
\left\langle\zeta_{a}^{\mu}(x, \mathbf{v}) \zeta_{b}^{\nu}\left(y, \mathbf{v}^{\prime}\right)\right\rangle= & (4 \pi)^{2} g^{2} \int d p d p^{\prime} d Q d Q^{\prime} p^{2} p^{\prime 2} \\
& \times Q_{a} Q_{b}^{\prime} v^{\mu} v^{\prime \nu}\left\langle\zeta(x, \mathbf{p}, Q) \zeta\left(y, \mathbf{p}^{\prime}, Q^{\prime}\right)\right\rangle \\
= & 2 \gamma T m_{D}^{2} v^{\mu} v^{\prime \nu} \mathcal{I}\left(\mathbf{v}, \mathbf{v}^{\prime}\right) \\
& \times \delta_{a b} \delta^{(4)}(x-y)
\end{aligned}
$$

The sum over the two helicities of the particles has been taken into account. In order to obtain Eq. (33), we have made 
use of Eqs. (19), (27) to (30), and of the relation $f_{\text {eq }}=$ $-T d f_{\text {eq }} / d p$ for the Maxwell-Boltzmann distribution.

The quantum plasma can be treated in exactly the same way. To confirm Eq. (33), we only need to take into account the change of normalization as commented above, and the relation $f_{\text {eq }}\left(1 \pm f_{\text {eq }}\right)=-T d f_{\text {eq }} / d p$ for the Bose-Einstein and Fermi-Dirac distributions, respectively.

Using the explicit expression for the collision integral and the stochastic noise it is possible to confirm the covariant conservation of the current, $D J=0[1,2]$.

We thus found that the correlator (33) is in full agreement with the result of $[1,2]$ for both the classical or the quantum plasma. While this correlator has been obtained in $[1,2]$ from the corresponding microscopic theory, here, it follows solely from the FDT. This way, it is established that the effective Boltzmann-Langevin equation found in [1] is indeed fully consistent with the fluctuation-dissipation theorem. More generally, the important observation is that the spectral functions as derived here from the entropy and the FDT do agree with those obtained in [2] from a microscopic phase space average. This guarantees that the formalism of [2] is consistent with the FDT.

In the above discussion we have considered the stochastic noise as Gaussian and Markovian. This is due to the fact that the small-scale fluctuations (those within a coarse-graining volume) are to leading order well separated from the typical relaxation scales in the plasma. Within the microscpoic approach in [2], these characteristics can be understood ultimately as a consequence of an expansion in a small plasma parameter (or a small gauge coupling). More precisely, the noise follows to be Gaussian when higher order correlators beyond quadratic ones can be neglected. The Markovian character of the noise follows because the ultra-soft modes are well separated from the soft ones, and suppressed in the collision integral to leading order. This way, the collision term and the correlator of stochastic noise are both local in $x$-space. Going beyond the logarithmic approximation, we expect from the explicit computation in [2] that the coupling of the soft and the ultra-soft modes makes the collision term non-local in coordinate space. This non-trivial memory kernel should also result in a non-Markovian, but still Gaussian, source for stochastic noise.

The present line of reasoning can in principle be extended to other approaches. Using the phenomenological derivation of Eq. (30) from [5], the same arguments as above justify the presence of a noise source with Eq. (33) in the corresponding Boltzmann equation [5-7]. It might also be fruitful to follow a similar line based on the entropy within a quantum field theoretical language. An interesting proposal to include selfconsistently the noise within a Schwinger-Dyson approach has been made recently in [10]. Along these lines, it might be feasible to derive the source for stochastic noise directly from the quantum field theory [6].

While we have concentrated the discussion on plasmas close to equilibrium, it is known that a fluctuation-dissipation theorem can be formulated as well for (stationary and stable) systems out-of-equilibrium [12]. It can also be extended to take non-linear effects into account [13]. Both the out of equilibrium situations and non-linear effects can be treated, in principle, with the general formalism presented in [2].

C.M. thanks the Institute for Nuclear Theory at the University of Washington for its hospitality and the Department of Energy for partial support during the completion of this work.
[1] D. Bödeker, Phys. Lett. B 426, 351 (1998); Nucl. Phys. B566, 402 (2000); B559, 502 (1999).

[2] D. F. Litim and C. Manuel, Phys. Rev. Lett. 82, 4981 (1999); Nucl. Phys. B562, 237 (1999).

[3] J. Jalilian-Marian, S. Jeon, R. Venugopalan, and J. Wirstam, hep-ph/9910299.

[4] R. D. Pisarski, hep-ph/9710370.

[5] P. Arnold, D. T. Son, and L. G. Yaffe, Phys. Rev. D 59, 105020 (1999); 60, 025007 (1999).

[6] J. P. Blaizot and E. Iancu, Nucl. Phys. B557, 183 (1999).

[7] M. A. Valle Basagoiti, U. Pais Vasco Report No. EHU-FT/ 9905, hep-ph/9903462.
[8] U. Heinz, Phys. Rev. Lett. 51, 351 (1983); Ann. Phys. (N.Y.) 161, 48 (1985); 168, 148 (1986).

[9] M. Bixon and R. Zwanzig, Phys. Rev. 187, 267 (1969).

[10] E. Calzetta and B. L. Hu, Phys. Rev. D 61, 025012 (2000).

[11] L. D. Landau and E. Lifshitz, Statistical Physics (Pergamon, Oxford, 1968), Pt I.

[12] Yu. L. Klimontovich, Statistical Physics (Harwood Academic, Chur, Switzerland, 1986); E. Lifshitz and L. Pitaevskii, Physical Kinetics (Pergamon, Oxford, 1981).

[13] A. G. Sitenko, Fluctuations and Non-Linear Wave Interactions in Plasmas (Pergamon, Oxford, 1982). 